Widefield ScIENCE AND TeChNOLOGY For the SKA

SKADS CONFERENCE 2009

S.A. Torchinsky, A. van Ardenne, T. van den Brink-Havinga, A.J.J. van Es, A.J. Faulkner (eds.)

4-6 November 2009, Château de Limelette, Belgium

\title{
RFI mitigation: cyclostationary criterion
}

\author{
R. Feliachi ${ }^{1}$, C. Dumez-Viou ${ }^{2}$, R. Weber ${ }^{1,2}$, and A.J. Boonstra ${ }^{3}$ \\ 1 Université d'Orléans, BP 6744, Orléans, Cédex 2, F-45067, France \\ 2 Observatoire de Paris, Station de radioastronomie, F-18330 Nançay, France \\ 3 ASTRON, Oude Hoogeveensedijk 4, 7991 PD Dwingeloo, P.O. Box 2, 7990 AA Dwingeloo, The Netherlands
}

\begin{abstract}
Radio astronomical observations are increasingly corrupted by radio frequency interferences. Thus, real-time filtering algorithms are becoming essential. One approach is to use a specific time property of the Telecoms signals : the cyclostationarity. This property can be exploited for detection purpose or filtering purpose. In particular, new generations of radio telescopes will be based on antenna arrays providing the possibility of applying spatial filtering techniques. In this paper, we compare the performance between classical approaches based on power statistics and cyclic approaches. This comparison is done through simulations on synthetic data and through simulations on real data acquired with the new generation low frequency array radio telescope, LOFAR.
\end{abstract}

\section{Introduction}

For several years, radio astronomy has had to face two contradictory trends. On the one hand, the exponential expansion of telecommunications has generated a growing demand on the electromagnetic spectrum, reducing the bandwidths available for good quality radio astronomical observations. On the other hand, radio astronomical needs in terms of sensitivity and bandwidth have also grown. As a result, radio frequency interference (RFI) mitigation has become a significant issue for current and future radio telescopes. The various methods that have been tried to limit RFI depend on the type of interference and the type of instruments. Time, frequency and/or spatial properties can be considered in order to find efficient excision processing techniques, see for example the inventory carries out in the framework of SKADS DS4T3 work package (Boonstra et al. 2009).

Most communication signals contain recurrent characteristics which stem, for example, from a transmitter carrier frequency or from a communication signal baud rate. Usually, these periodicities are scrambled and hidden by the intrinsic signal randomness. However, to some extent, these periodicities can be regenerated. If it is the case, such signals are so called cyclostationary signals, or in short, cyclic signals. This specific property can be used to discriminate them from natural signals.

\section{What is the cyclostationarity?}

Mathematically, a cyclostationary process means that its statistics are periodic with time. For example, let us consider the second order statistics given by the correlation of a given process $x(t)$ :

$$
R(t, \tau)=E\left\{x\left(t-\frac{\tau}{2}\right) x\left(t+\frac{\tau}{2}\right)\right\}
$$

where $E\{$.$\} represents the ensemble average operator.$

If the process is modeled as stationary then $R(t, \tau)=R(\tau)$. If the process is modeled as cyclostationary then $\exists T / R(t+T, \tau)=$
$R(t, \tau)$. T is called the cyclic period. Review papers on cyclo stationarity can be found in Gardner Gardner et al. (2006) or SerpedinSerpedin et al. (2005).

To illustrate these concepts, let us consider the following simple baseband signal, $x(t)=\sum_{k \in Z} a_{k} g(t-k T)$, where $a_{k}$ is a random digital message with power $\sigma_{a}^{2}, g(t)$, its pulse shape and $T$, its baud rate. The correlation of $x(t)$ becomes :

$$
R(t, \tau)=\sigma_{a}^{2} \sum_{k \in Z} g\left(t-k T+\frac{\tau}{2}\right) g\left(t-k T-\frac{\tau}{2}\right)
$$

One can easily verify that $R(t, \tau)$ is $T$-periodic. So, it can be decomposed in Fourier series and the corresponding Fourier coefficients are $(k \in Z)$ :

$$
R^{\alpha=\frac{k}{T}}(\tau)=\frac{\sigma_{a}^{2}}{T} \underbrace{\int_{-\infty}^{+\infty} g\left(t+\frac{\tau}{2}\right) g\left(t-\frac{\tau}{2}\right) \exp (-j 2 \pi \alpha t) d t}_{r_{g}^{\alpha}(\tau)}
$$

In practice, a time average approach is preferred and $R^{\alpha}(\tau)$, also called the cyclic correlation, is given by:

$$
R^{\alpha}(\tau)=\lim _{N \rightarrow+\infty} \frac{1}{N} \int_{N} x\left(t+\frac{\tau}{2}\right) x\left(t-\frac{\tau}{2}\right) \exp (-j 2 \pi \alpha t) d t
$$

If $R^{\alpha}(\tau)$ is non-zero for some cyclic frequencies, $\alpha$, with $\alpha \neq$ 0 , then $x(t)$ can be modeled as cyclostationary. Note that for $\alpha=0$, we retrieve the expression of the classical correlation. Figure 1 gives the plots of these previous expressions for the considered example with a rectangular pulse function.

In the cyclostationary case, the temporal periodicity of the correlation can be exploited to extract RFI information from the noise. In the next two sections, two RFI mitigation techniques based on the exploitation of the cyclostationarity are presented.

\section{Example of cyclostationary detection}

We suppose now that $x(t)$ is a mix of a stationary signal (i.e. a cosmic source and/or the system noise) and a cyclostationary signal (i.e. a RFI). Let us consider the following criterion: 

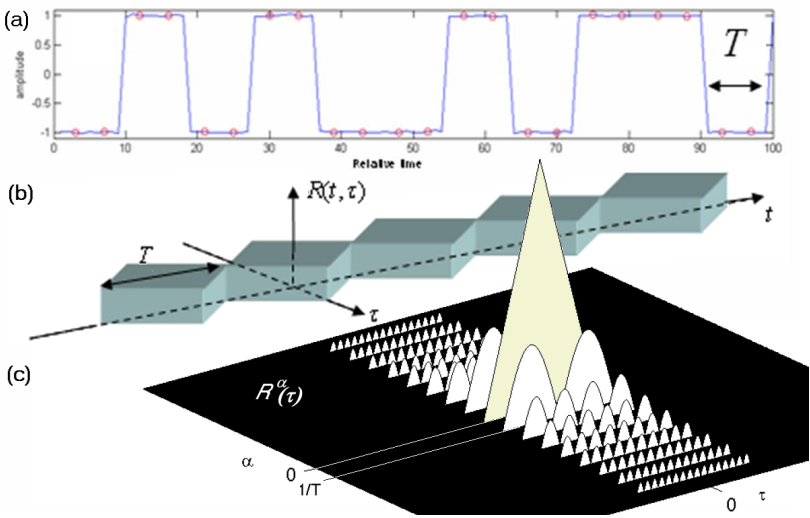

Fig. 1: Random binary signal, $x(t)=\sum_{k \in Z} a_{k} g(t-k T)$ with $g(t)$ rectangular. (a) Temporal view. (b) correlation of $x(t) . R(t, \tau)$ is $T$-periodic. (c) the cyclic correlation $R^{\alpha}(\tau) . R^{\alpha}(\tau) \neq 0$ for $\alpha=\frac{k}{T}, k \in Z$. A stationary process will provide information only at $\alpha=0$.

$$
C_{N}^{\alpha}=\frac{1}{N} \sum_{n=0}^{N-1}|x|^{2}(n) \exp (-j 2 \pi \alpha n)
$$

This criterion is just derived from the cyclic correlation defined in Eq. 4 by considering 1) discrete samples and 2) a finite averaging 3) for $\tau=0$. Actually, through this criterion, we are just looking for periodicities in the instantaneous power fluctuations. To make this detector robust again slow power variations, we define a normalized version of our previous criterion:

$$
D_{N}^{\alpha}=\frac{\sqrt{N} C_{N}^{\alpha}}{C_{N}^{0}}
$$

In Weber (Weber et al. 2007), the statistical properties of this detector have been derived as a function of the interference to noise ratio. Figure 2 plots the results obtained with an AM signal. If the expected cyclic frequency is not known, a blind cyclostationary detection can be perform by Fourier transform of the instantaneous power. Once spectral lines are detected at non-zero frequencies, it is the signature of a cyclostationary signal.

From that consideration, an operational cyclic detector has been implemented on a real time digital backend at Nançay Observatory. The backend is described in Figure 3.a. The algorithm is implemented into a digital programmable component Virtex II, a FPGA from the Xilinx company. The successive steps are:

1. Channelization of the signal coming from the radio telescope. The signal in each channel is supposed to be complex. This process is done in real time by the digital receiver.

2. To reduce the computational load of the cyclic detector, the algorithm is applied to the real part only $\left(s_{r}(n)\right)$ of the signal.We compute the Fourier transform, $F F T_{N}^{m}(f)$, over $N$ samples on $s_{r}^{2}(n)$ for the $m^{\text {th }}$ channel, $m=1, \ldots, M$. $M$ is the number of channels.

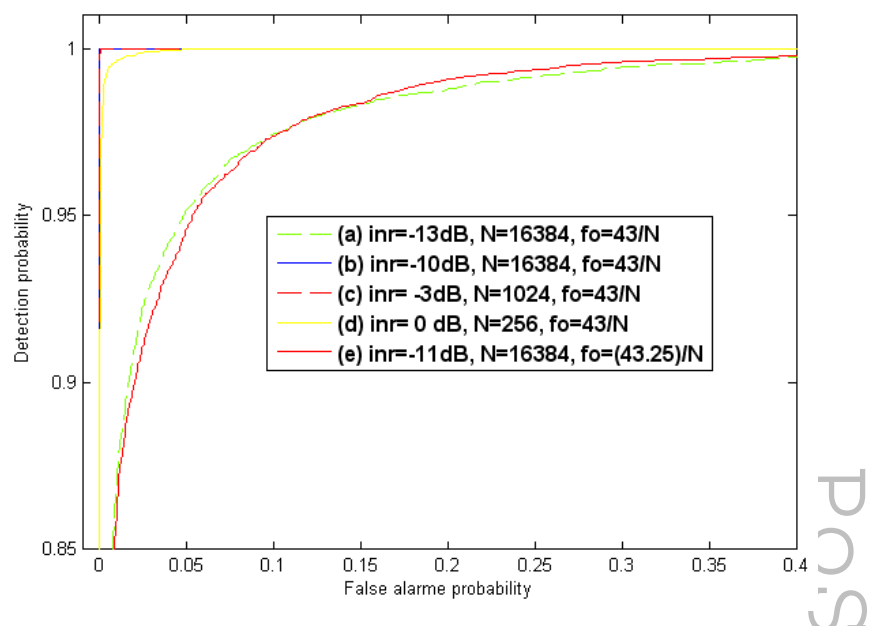

Fig. 2: Probability of detection vs. probability of false alarm for the cyclostationary detector (Eq. 6). The RFI is an AM modulation. Its carrier frequency is $f_{o}$ and the corresponding expected cyclic frequency is $\alpha=2 f_{o}$. This simulation is based on 10000 runs. In cases (a) to (d), $f_{o}$ is chosen so that $2 f_{o}$ is multiple of $1 / N$ (i.e. the $2 f_{o}$ spectral line is in the middle of the $N$ bin FFT channel, no attenuation). In case (e), the $2 f_{0}$ spectral line is between two $N$ bin FFT channels, which is the worst case. The channel attenuation at Nyquist frequency for a rectangular window is $\approx 2 d B$. Thus, (e) should be similar to (a)

3. According to a given threshold $\xi$ derived from the theoretical study, we will consider that a RFI is present on the $m^{\text {th }}$ channel if:

$$
\begin{aligned}
& \exists k>0 / \frac{\sqrt{N}\left|F F T_{N}^{m}(k)\right|}{F F T_{N}^{r}(0)} \geq \xi \\
& \text { with } \xi=\sqrt{-2 \log (\mathrm{pfa})}
\end{aligned}
$$

where pfa is the expected probability of false alarm.

Figure 3.b shows some results obtained in the decameter band. In the next section, the cyclostationary concept will be extended to correlation matrices by considering radio telescope arrays.

\section{Example of cyclostationary spatial processing}

Consider a telescope array consisting of $p$ antennas, each having a received signal $x_{k}(t), k=1, \ldots p$ (see Figure 4.a). It is assumed that the narrowband condition holds and that geometric delays for each antenna and each impinging source can be represented by phase shifts. In this case, the telescope correlation matrix $\mathbf{R}$ can be modeled as:

$$
\begin{aligned}
\mathbf{R} & =E\left\{\mathbf{x} \mathbf{x}^{H}\right\} \\
& =\mathbf{A}_{r} \mathbf{R}_{r} \mathbf{A}_{r}^{H}+\mathbf{A}_{s} \mathbf{R}_{s} \mathbf{A}_{s}^{H}+\mathbf{N}
\end{aligned}
$$

where (.) ${ }^{H}$ is the conjugate transpose operator, $\mathbf{R}_{r}$ is the $K_{r} \times K_{r}$ correlation matrix due to the $K_{r} \alpha$-cyclostationary sources (i.e. the RFI), $\mathbf{R}_{s}$ is the $K_{s} \times K_{s}$ correlation matrix due to the $K_{s}$ others sources (i.e. stationary sources and non $\alpha$-cyclostationary RFI if any) and $\mathbf{N}$ is the $p \times p$ correlation matrix due to the system noise. These matrices contain the signal information. 

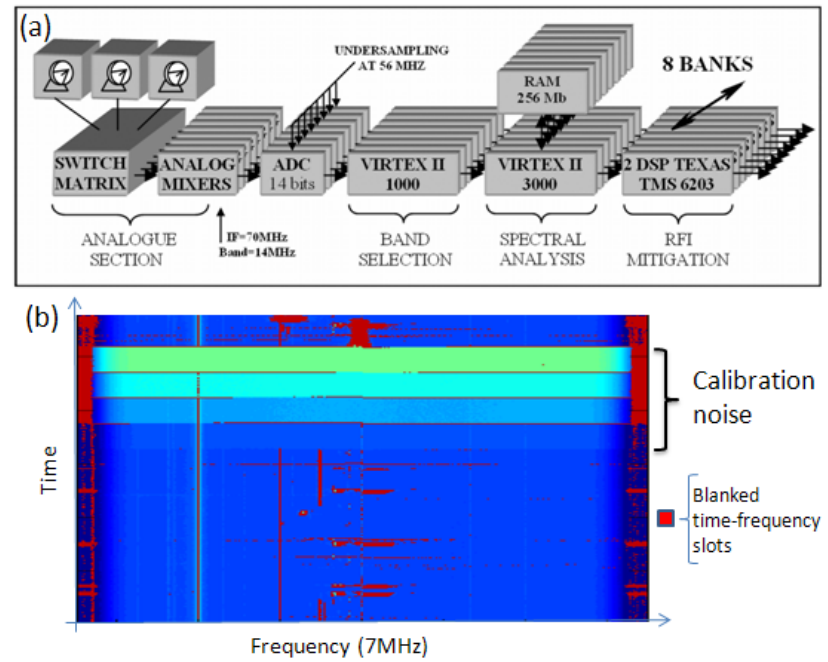

Fig. 3: a) The current functionalities of the digital system are high dynamic range of $70 \mathrm{~dB}$, bandwidth selection facilities ranging from $875 \mathrm{kHz}$ to $14 \mathrm{MHz}$, high spectral resolution through a polyphase filter bank with up to 8192 channels with 49152 coefficients. A more complete description can be found in Weber (Weber et al. 2005) b) Results obtained in the decameter band. The total bandwidth is $7 \mathrm{MHz}$. The number of channels is $M=2048$. On each channel, $N=2048$ samples are used to compute the criterion. The cyclic detector is robust against power fluctuations generated by the successive calibration noise diode pulses added to the signal.

Matrices $\mathbf{A}_{r}\left(p \times K_{r}\right)$ and $\mathbf{A}_{s}\left(p \times K_{s}\right)$ contain the spatial signatures of the impinging sources.

In order to remove RFI from the received data, we can filter them out by applying a spatial null in the direction of the undesired signals. Each received signal is identified by its spatial signature in the received data model. If the information contained in $\mathbf{A}_{r}$ can be estimated, then the RFI can be filtered out (see Figure 4.b).

The proposed estimation method is based on subspace decomposition. If the cosmic sources are negligible and the system noise is calibrated (i.e. $\mathbf{R} \approx \mathbf{A}_{r} \mathbf{R}_{r} \mathbf{A}_{r}^{H}+\sigma^{2} \mathbf{I}$ where $\mathbf{I}$ is the identity matrix and $\sigma^{2}$ the system noise power ), Boonstra (2005) has demonstrated that a close estimate of the information contained in $\mathbf{A}_{r}$ can be derived from the eigenvalue decomposition (EVD) of $\mathbf{R}$ (see Figure 4.c).

The same idea can be applied on the cyclic correlation matrix defined by either its ensemble average version or its time average version

$$
\begin{gathered}
\mathbf{R}^{\alpha}=E\{\mathbf{R}(t) \exp (-2 \pi \alpha t\} \\
\mathbf{R}^{\alpha}=\lim _{N \rightarrow+\infty} \frac{1}{N} \int_{N} \mathbf{x}(t) \mathbf{x}^{H}(t) \exp (-j 2 \pi \alpha t) d t
\end{gathered}
$$

where $\mathbf{R}(t)$ is the instantaneous correlation matrix.

The great interest of the cyclic approach is that $\mathbf{R}^{\alpha}$ is asymptotically RFI-only dependent. Indeed, it can be easily shown that:

$$
\mathbf{R}^{\alpha}=\mathbf{A}_{r} \mathbf{R}_{r}^{\alpha} \mathbf{A}_{r}^{H}
$$

where $\mathbf{R}_{r}^{\alpha}$ is the RFI cyclic correlation matrix.
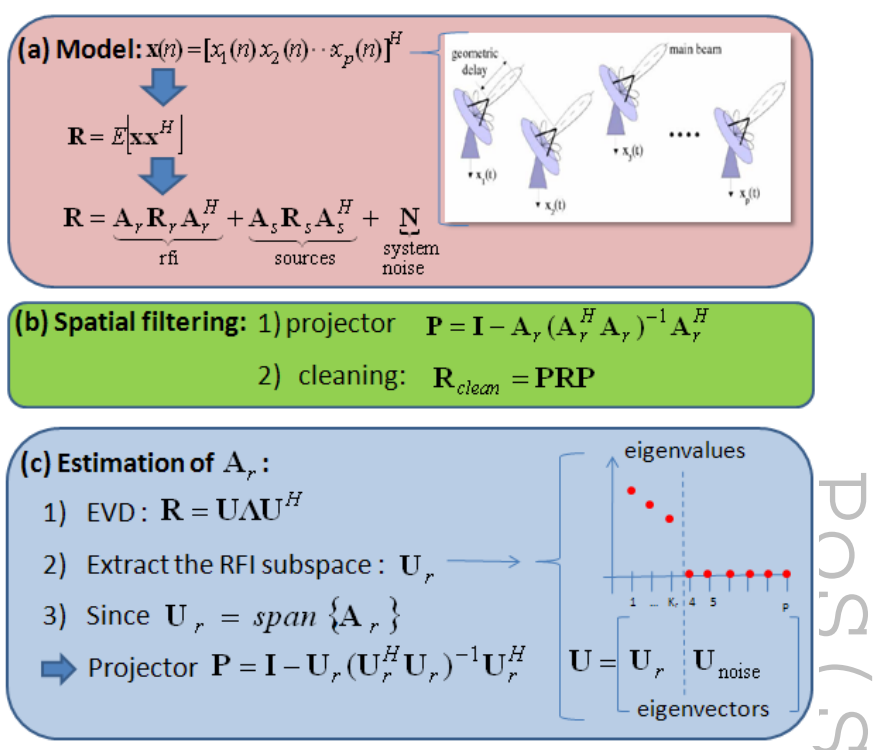

Fig. 4: a) The data array model: The correlation matrices $\mathbf{R}_{r}, \mathbf{R}_{s}$ and $\mathbf{N}$ contain the signal information. Matrices $\mathbf{A}_{r}$ and $\mathbf{A}_{s}$ contain the spatial signatures of the impinging sources. b) Spatial filtering: This method is based on the estimation of the RFI spatial signature vector, $\mathbf{A}_{r}$, from the correlation matrix, $\mathbf{R}$ followed by a subspace projection to remove that dimension from the correlation matrix, $\mathbf{R}$. To preserve the integrity of the cosmic information, $\mathbf{A}_{s} \mathbf{R}_{s} \mathbf{A}_{r}^{H}$, a correction matrix must be applied on the cleaned correlation matrix, $\mathbf{R}_{\text {clean }}$ (see Boonstra Boonstra 2005). c) Estimation of $\mathbf{A}_{r}$ : the signal subspace, $\mathbf{U}_{r}$, (i.e. subspace formed by the eigenvectors associated to the $K_{r}$ largest eigenvalues) will span the same dimensions as the RFI spatial signature matrix, $\mathbf{A}_{r}$, assuming that all other signal contributions in the matrix correlation model are negligible.

Thus, by using $\mathbf{R}^{\alpha}$ rather than $\mathbf{R}$, the RFI spatial signature estimation is more robust.

Remark: It is assumed that the $K_{r}$ RFI sources have the same cyclostationary property. If not, the algorithm will be applied on each group of RFI. More results can be found in Feliachi et al. (2009).

We have applied the classic (i.e. using $\mathbf{R}$ ) and cyclic (i.e. using $\mathbf{R}^{\alpha}$ ) spatial filtering to real observations acquired with the LOFAR radio telescope. LOFAR is a phased array interferometric telescope developed by ASTRON in the Netherlands. It is currently in the roll-out phase and operates in the band 30$240 \mathrm{MHz}$. In LOFAR, antennas are grouped in so-called stations in which the signals of 100 antennas are combined using phased-array beamforming. The beamformed signals of many stations are combined centrally by correlating them. Sky images are produced by inverse Fourier transforming the correlation products. We have observed in the 160-240 MHz LOFAR band, which contains a very strong transmitter (pager) at 170 $\mathrm{MHz}$ with an INR of $47 \mathrm{~dB}$. The array configuration consisted of $\mathrm{M}=8 \mathrm{LOFAR}$ antennas.

Figure 5 shows the eigenvalues obtained from the classic and the cyclic correlation matrix which were derived from baseband data of the antennas of one station. The cyclic frequency, $\alpha$, of the pager has been first estimated from the data: 


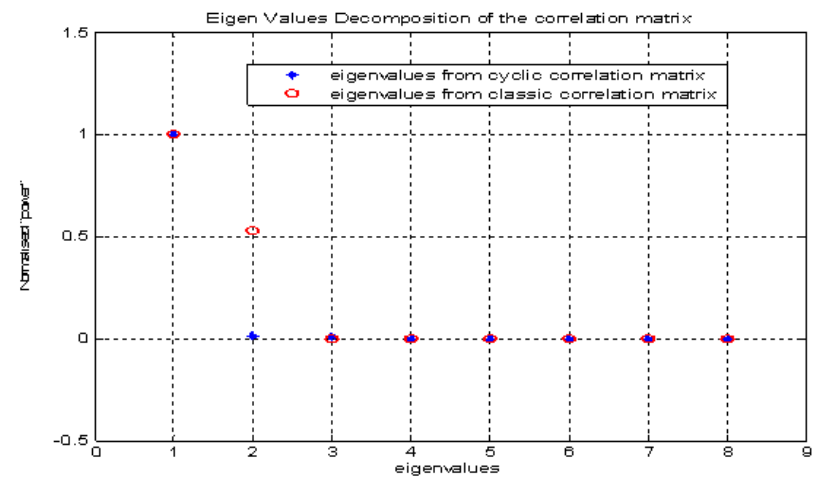

Fig. 5: The eigenvalue decomposition of the classic and the cyclic correlation matrix estimated from real data acquired with the LOFAR telescope. A strong transmitter is present in the dataset (see Figure 6). $p=8$ antennas have been used and the correlation matrices have been estimated over $N=65536$ samples.

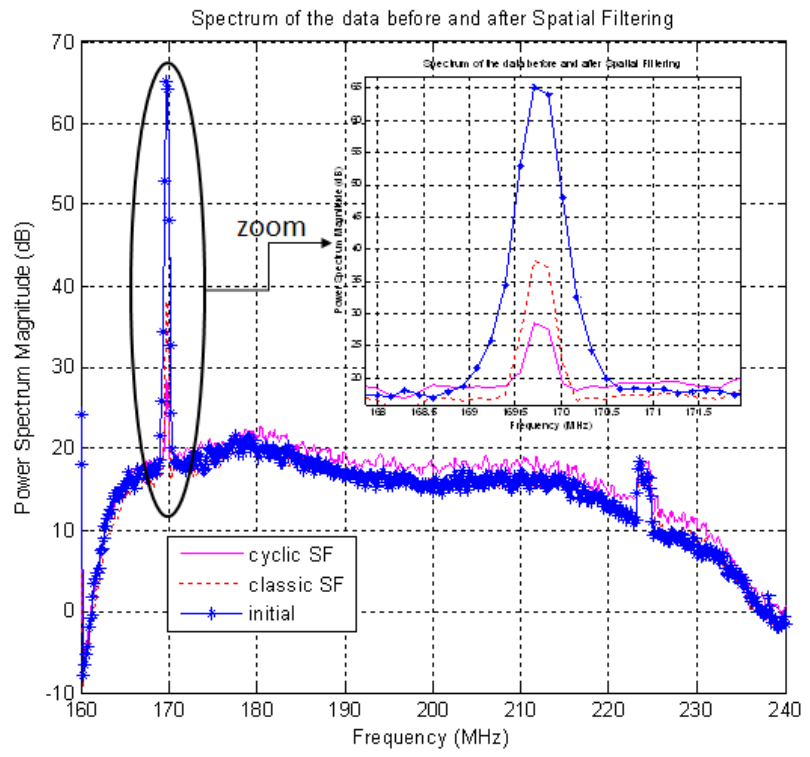

Fig. 6: Spectrum of one antenna output after applying cyclic and classic spatial filtering $(p=8, N=65536)$.

$\alpha=0.1221$ in normalized frequency. The figure shows that the interferer signal subspace can be fairly well estimated using one dimension in the cyclic decomposition, whereas it needs two dimensions in the classic one. The more dimensions are used to remove the interferer, the more information about the cosmic sources is thrown away as well. We used therefore only the eigenvector corresponding to the strongest eigenvalue to build the projector for both methods. Figure 6 shows the effect of the projector on the pager. Using the cyclic method, the pager is removed more effectively compared with the classic approach.

\section{Conclusions}

In this paper, we have described two RFI mitigation approaches based on the cyclostationary properties of the RFI. The first method is a blind cyclostationary detector which has been implemented into a real time receiver. The second one is a cyclic spatial filtering. These methods seem to be an attractive alternative to the classic method based on power statistics. Indeed, as shown with simulations and experimental results, it leads to interesting performances for cases where there are relatively strong cosmic sources or for cases where the input signals are uncalibrated. Feliachi (2010) will describe in her upcoming $\mathrm{PhD}$ manuscript other applications of cyclostationarity for phased array radio telescopes.

Acknowledgements. The authors would like to thank Pierre Colom, DS4-T3 task leader, Observatoire de Paris, France, Philippe Ravier and Rachid Harba, both from the University of Orléans, France, for supplying them with helpful materials and remarks. The authors also want to thank the European Commission Framework Program 6, Project SKADS (contract no 011938), the Région Centre, the French research consortium GDR-ISIS and the Dutch Helena Kluyver female visitor programme for funding part of this work.

\section{References}

Boonstra, A. J. 2005, Radio Frequency Interference Mitigation in Radio Astronomy, Thesis, Delft university, The Netherlands.

Boonstra, A. J., Weber, R. eds. 2009, RFI Mitigation Methods Inventory, SKADS DS4T3 Report

Feliachi, R., Weber, R., Boonstra, A. J. 2009, Cyclic spatial filtering in radio astronomy : application to LOFAR data (Eusipco'2009), Glasgow, Scotland.

Feliachi, R. in preparation, Spatial processing of cyclostationary interferers for phased arrays radio telescopes, Thesis, University of Orléans, France

Gardner, W.A, Napolitano, A.\& Paura, L. 2006 , Cyclostationarity: Half a century of research (Signal Processing), 4,86

Serpedin, E., Panduru, F., Sari, I. \& Giannakis, G. B. 2005, Bibliography on cyclostationarity (Signal Processing), 12, 85

Weber, R., Viou, C., Coffre, A., Denis, L., et al. 2005,DSP Enabled Radio Astronomy: Towards IIIZW35 Reconquest (JASP), 16

Weber, R., Zarka, P., Ryabov, V. B., Feliachi, R., et al. 2007, Data preprocessing for decametre wavelength exoplanet detection: an example of cyclostationary rfi detector (Eusipco'2007), Poznan, Poland. 